\title{
Core/Shell and Hollow Polymeric Capsules Prepared from Calcium Carbonate Whisker
}

\author{
Takashi SASAKI, ${ }^{\dagger}$ Takayuki KITAGAwA, Shintaro SATO, \\ Satoshi IRIE, and Kensuke SAKURAI \\ Department of Materials Science \& Engineering, Fukui University, 3-9-1 Bunkyo, Fukui 910-8507, Japan
}

(Received January 17, 2005; Accepted March 12, 2005; Published June 15, 2005)

\begin{abstract}
Polymeric capsules of a rod-like shape were prepared by depositing a poly(methyl methacrylate) or polystyrene thin layer onto the surface of calcium carbonate whisker via emulsion polymerization. The obtained core/ shell capsules were also converted to hollow capsules by dissolving the core material in a hydrochloric acid solution. The average thickness of the outer shell (polymer layer) ranged from 29 to $72 \mathrm{~nm}$. The glass transition temperatures $T_{\mathrm{g}}$ 's of the outer shell were almost identical to those of the bulk both for the core/shell and hollow capsules in contrast to the results for ultrathin polymer films, which have been reported to show significantly different $T_{\mathrm{g}}$ from the bulk value. [DOI 10.1295/polymj.37.434]

KEY WORDS Polymeric Capsules / Calcium Carbonate Whisker / Glass Transition / Emulsion Polymerization /
\end{abstract}

Recently, development of nano-sized capsules and core/shell particles is a topic of growing interest in nanotechnology and in biomedical applications to such as drug delivery system, hyperthermia with nano-sized magnetic particles, and so forth. Extensive studies have been done to fabricate core/shell and hollow capsules by using various techniques such as micellar formation of block copolymers, ${ }^{1,2}$ seed emulsion polymerization, ${ }^{3,4}$ and layer-by-layer deposition of polyelectrolytes. ${ }^{5,6}$ On the other hand, the dynamics of nano-sized polymers have been revealed to be quite different from that of a bulk material, which may be due to anomalous dynamics near an interface and/or to the confinement effects. ${ }^{7-9}$ Moreover, the thermal property of nano-sized polymers has been revealed to depend on their geometry: the glass transition temperature $T_{\mathrm{g}}$ of a polymer thin film of which the thickness is less than $100 \mathrm{~nm}$ depends significantly on the film thickness, ${ }^{9-11}$ while microspheres of polystyrene of which the diameter is $40-500 \mathrm{~nm}$ exhibit a $T_{\mathrm{g}}$ almost identical to the bulk $T_{\mathrm{g}}{ }^{12,13} \mathrm{It}$ is intriguing and important to investigate thermal properties of nanosized particles with various geometrical shapes such as non-spherical capsules.

In this study, we prepared novel core/shell and hollow capsules using calcium carbonate whisker with a rod-like shape as a template. Emulsion polymerization of methyl methacrylate or styrene was performed in the presence of calcium carbonate whisker particles, whereby thin polymer layer of poly(methyl methacrylate) (PMMA) or polystyrene (PS) was deposited onto the surface of the calcium carbonate whisker particles. ${ }^{14}$ The obtained core/shell particles were further converted to hollow particles by removing the core (calcium carbonate) by treating with hydrochloric acid. The morphology and thermal property of the samples thus prepared were investigated by transmission electron microscopy (TEM) and differential scanning calorimetry (DSC).

\section{EXPERIMENTAL}

\section{Preparation of Polymeric Capsules}

Methyl methacrylate and styrene were distilled under reduced pressure. Calcium carbonate whisker (BS-P) was supplied by Maruo Calcium Co. The whisker was treated with phosphoric acid to produce very thin layers of calcium phosphate on the surface; the diameter of the particles ranged from 300 to $600 \mathrm{~nm}$, and their length ranged from 1.0 to $3.0 \mu \mathrm{m}$. First, a monomer emulsion mixture was prepared which consisted of $15 \mathrm{~g}$ of monomer (methyl methacrylate or styrene), $0.14 \mathrm{~g}$ of laurylbenzenesulfonic acid sodium salt, $0.47 \mathrm{~g}$ of 2-hydroxymethyl methacrylate, $0.04 \mathrm{~g}$ of methacrylic acid 3-(trimethoxysilyl)propyl ester, ${ }^{15}$ and $3.1 \mathrm{~g}$ of distilled water. Into a four-necked flask was placed $12 \mathrm{~g}$ of BS-P, $0.228 \mathrm{~g}$ of laurylbenzenesulfonic acid sodium salt, and $200 \mathrm{~g}$ of distilled water, and the mixture was heated to $80^{\circ} \mathrm{C}$ with stirring at $400 \mathrm{rpm}$. Emulsion polymerization was then started by adding peroxodisulfate (1.0$1.5 \mathrm{~mL}$ of a $10 \mathrm{wt} \%$ aqueous solution) and the monomer emulsion mixture prepared above very slowly to the flask for $45 \mathrm{~min}$ under constant stirring ( $400 \mathrm{rpm}$ ) at $80^{\circ} \mathrm{C}$. The reaction proceeded further for $3 \mathrm{~h}$ at $80^{\circ} \mathrm{C}$, and then, the mixture was cooled to $55^{\circ} \mathrm{C}$,

${ }^{\dagger}$ To whom correspondence should be addressed (E-mail: sasaki@matse.fukui-u.ac.jp). 
followed by adding a very small amount of sodium formaldehydesulfoxylate in order to complete the polymerization reaction. After the solution was cooled to $25^{\circ} \mathrm{C}$, particles of BS-P whisker coated by polymerized material (core/shell particles) precipitated spontaneously, while polymer particles (microspheres) without the BS-P core still remained suspended. The precipitate was separated carefully by filtration, washed with distilled water several times, and dried in vacuum for $72 \mathrm{~h}$ at room temperature.

The hollow capsules were prepared in the following manner: $3.0 \mathrm{~g}$ of the powder of core/shell particles prepared above was dispersed in $75 \mathrm{~g}$ of distilled water by gentle sonication, and then, hydrochloric acid was added with stirring to dissolve the core material completely until the $\mathrm{pH}$ value became less than 1.0. The mixture was further stirred for $2 \mathrm{~h}$, and insoluble material was separated by filtration. The separated material was then washed with distilled water several times until the $\mathrm{pH}$ value of the filtrate became 7.0. The yielded hollow capsules were then dried in vacuum for $72 \mathrm{~h}$ at room temperature. As reference bulk samples, cast films were prepared from $5 \mathrm{wt} \%$ chloroform solutions of the hollow samples. The films were dried under vacuum at $80^{\circ} \mathrm{C}$ for $48 \mathrm{~h}$. Molecular weights of the polymerized materials were measured by size exclusion chromatography using two $30 \mathrm{~cm}$ columns (TOSOH G2500 and G300), and the eluent was THF (flow rate: $1 \mathrm{~mL} \mathrm{~min}^{-1}$ ).

\section{Analysis}

Morphology and sizes of the obtained polymeric capsules were examined by using a transmission and scanning electron microscope Jeol JEM-2000FXII. Here, the samples were observed directly on microgrids without any shadowing. We also performed wide-angle X-ray diffractometry (WAXD) for the obtained polymeric capsules by using a Rigaku RINT2100 equipped with a $2 \mathrm{~kW} \mathrm{Cu} \mathrm{K \alpha}$ source. DSC measurements were done by using a power compensation type calorimeter PerkinElmer Pyris Diamond. The temperature and heat flow were calibrated with an indium standard. An ice-water bath was used as a cooling system. All measurements were done in a nitrogen atmosphere, and typical sample size was 12-20 mg for the core/shell samples and 5-7 mg for the hollow samples. The heating and cooling scans were done at a scanning rate of $10 \mathrm{~K} \mathrm{~min}^{-1}$. We also performed DSC measurements of step-scan mode, which provides information essentially equivalent to that obtainable by temperature-modulated DSC. ${ }^{16}$ From the step-scan measurements, we evaluated the thermodynamic effect (reversing specific heat) and kinetic effect such as the hysteresis behavior (enthalpy recovery at around $T_{\mathrm{g}}$ ). In the step-scan measure-
Table I. Molecular weights of the polymers deposited on the surface of BS-P

\begin{tabular}{lcc}
\hline & $\begin{array}{c}M_{\mathrm{n}} \\
\left(\mathrm{kg} \mathrm{mol}^{-1}\right)\end{array}$ & $\begin{array}{c}M_{\mathrm{w}} \\
\left(\mathrm{kg} \mathrm{mol}^{-1}\right)\end{array}$ \\
\hline PMMA & 28 & 173 \\
PS & 10 & 36 \\
\hline
\end{tabular}

ments, we repeated a $2 \mathrm{~K}$ step of heating that was achieved with a rate of $5 \mathrm{~K} \mathrm{~min}^{-1}$ followed by a temperature holding segment. Detailed conditions for the step-scan measurements were described elsewhere. ${ }^{17}$

\section{RESULTS AND DISCUSSION}

Table I shows the molecular weights for the polymerized materials prepared in this study. The molecular weights are lower and their distributions are broader (especially for PMMA) than those reported for usual emulsion polymerization and theoretical predictions. ${ }^{18,19}$ The probability of bimolecular reaction between radicals may be enhanced for our system compared with the polymerization in small micelles (usual emulsion polymerization): in the former system, polymerization proceeds in a relatively large volume near the surface of the whisker.

Figures 1 and 2 show electron micrographs for the prepared polymeric capsules of PMMA and PS, respectively. The core part of the core/shell particles (PMMA/BS-P and PS/BS-P) are contrasted as dark parts due to the presence of calcium atoms. We see that the surface of BS-P particle is covered with the polymer fairly well for both PMMA and PS samples. This was also confirmed by the DSC results as will be described later. Yielding such core/shell particles suggests that in the emulsion, BS-P particles were preferably incorporated inside the micelle together with the monomer. As for the hollow samples, we see no dark core material inside the particles. WAXD profiles for these hollow samples revealed no diffraction of calcium carbonate crystallite, thus, we confirmed that the core part was removed. This is also supported by the DSC result ( $\Delta C_{p}$ values) as will be shown later. Figure 3 shows scanning electron micrographs for the obtained hollow capsules. Clearly we can see a rod-like shape, which indicates that the hollow capsules have not been collapsed after the removal of the core.

The diameter of the obtained capsules and the thickness of the outer shell (polymer thin layer) are listed in Table II. The average values reported in this table were obtained by sampling of a few hundreds of particles. The values of the standard deviations for both the diameter $d$ and thickness of the outer polymer shell $l$ are quite large indicating that considerable 
(a)

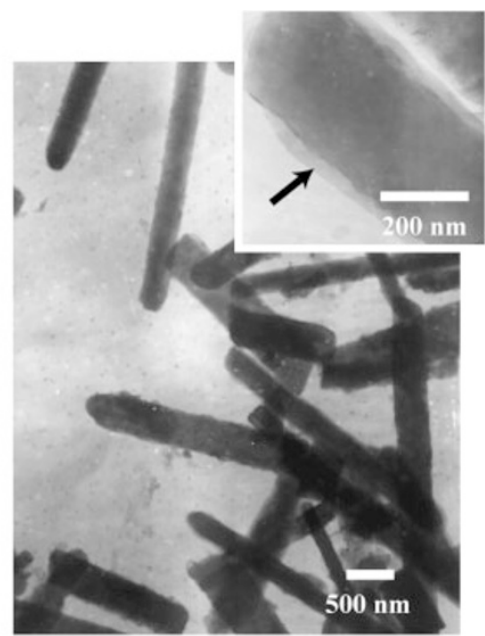

(b)

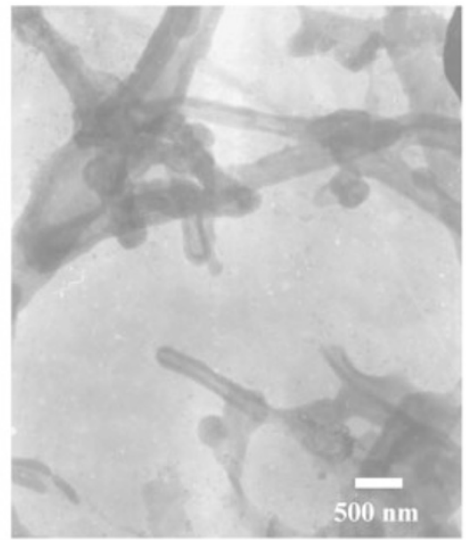

Figure 1. Transmission electron micrographs for (a) PMMA/ BS-P core/shell capsules, and (b) PMMA hollow capsules. The shell part (PMMA) is pointed by an arrow in (a).

distributions exist. We can see that for all the samples, $l$ is less than $100 \mathrm{~nm}$. Note that the diameter for the core/shell capsules is greater than that for the corresponding hollow capsules. This suggests that some structural relaxation or stress release in the polymeric layer occurred when the core material was removed. Note also that $l$ increases when the core material is removed. This may be explained qualitatively by a simple geometric postulate that the smaller the diameter of a tube is, the larger must be its thickness, if the total mass is preserved. However, the data listed in Table II are not quantitatively consistent with the above consideration, i.e., the expected $l$ values are $54 \mathrm{~nm}$ for PMMA hollow and $69 \mathrm{~nm}$ for PS hollow. This may be due to the large distributions in sizes as indicated by the large standard deviation values.

Table III shows $T_{\mathrm{g}}$ and $\Delta C_{p}$ values obtained from the step-scan mode of DSC. $T_{\mathrm{g}}$ was evaluated as the mid-point temperature, and $\Delta C_{p}$ was evaluated as the heat capacity jump at the evaluated $T_{\mathrm{g}}$. The standard deviations for $T_{\mathrm{g}}$ and $\Delta C_{p}$ values in this table were estimated to be at most $2.0 \mathrm{~K}$ and $0.02 \mathrm{~J} \mathrm{~g}^{-1} \mathrm{~K}^{-1}$, (a)

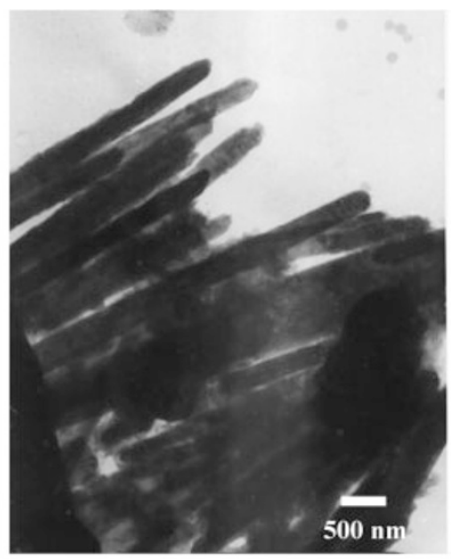

(b)

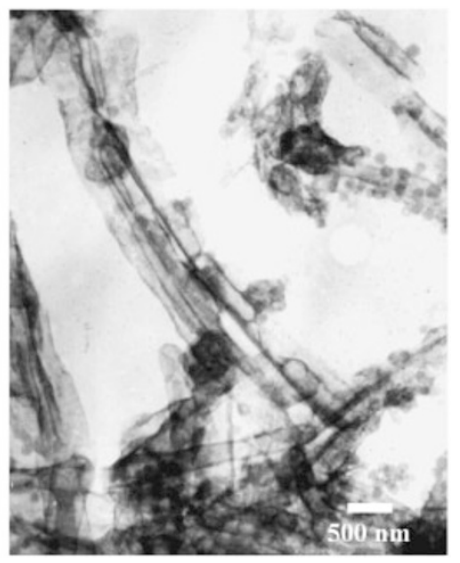

Figure 2. Transmission electron micrographs for (a) PS/BS-P core/shell capsules, and (b) PS hollow capsules.

(a)

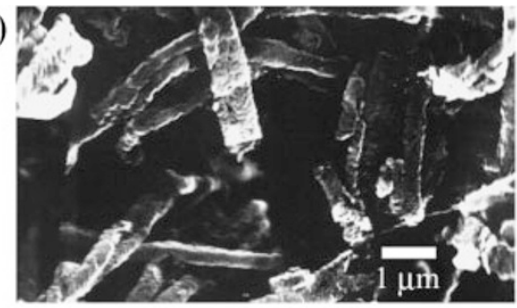

(b)

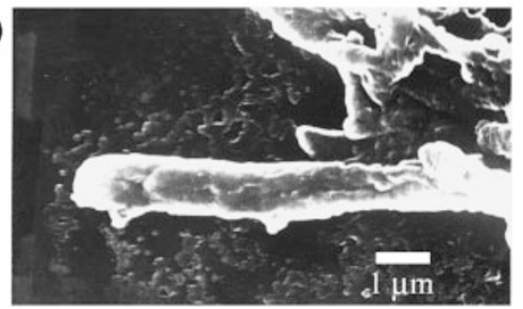

Figure 3. Scanning electron micrographs for (a) PMMA hollow capsules, and (b) PS hollow capsules.

respectively, by executing multiple measurements. PMMA/BS-P and PMMA hollow show $T_{\mathrm{g}}$ values very similar to the bulk value, and this is also the case for PS/BS-P and PS hollow samples. Note also that 
Table II. Diameter of the polymeric capsules and thickness of the outer shell

\begin{tabular}{lcccc}
\hline & $\begin{array}{c}d^{\mathrm{a}} \\
(\mathrm{nm})\end{array}$ & $\begin{array}{c}\sigma_{d}{ }^{\mathrm{b}} \\
(\mathrm{nm})\end{array}$ & $\begin{array}{c}l^{\mathrm{c}} \\
(\mathrm{nm})\end{array}$ & $\begin{array}{c}\sigma_{l}{ }^{\mathrm{d}} \\
(\mathrm{nm})\end{array}$ \\
\hline PMMA/BS-P & 414 & 118 & 38 & 15 \\
PMMA Hollow & 322 & 112 & 72 & 32 \\
PS/BS-P & 443 & 154 & 29 & 11 \\
PS Hollow & 244 & 41 & 33 & 9 \\
\hline
\end{tabular}

${ }^{\mathrm{a}}$ Diameter of the capsule including outer shell. ${ }^{\mathrm{b}}$ Standard deviation for $d .{ }^{\mathrm{c}}$ Thickness of the outer shell. ${ }^{\mathrm{d}}$ Standard deviation for $l$.

Table III. $T_{\mathrm{g}}$ and $\Delta C_{p}$ values obtained from the step-scan mode of DSC

\begin{tabular}{lcc}
\hline & $\begin{array}{c}T_{\mathrm{g}} \\
\left({ }^{\circ} \mathrm{C}\right)\end{array}$ & $\begin{array}{c}\Delta C_{p} \\
\left(\mathrm{~J} \mathrm{~g}^{-1} \mathrm{~K}^{-1}\right)\end{array}$ \\
\hline PMMA/BS-P & 122 & 0.087 \\
PMMA Hollow & 121 & 0.290 \\
PMMA Bulk & 120 & 0.280 \\
PS/BS-P & 101 & 0.036 \\
PS Hollow & 99 & 0.283 \\
PS Bulk & 97 & 0.289 \\
\hline
\end{tabular}

$\Delta C_{p}$ values of the hollow samples are almost identical to their bulk values, indicating that the core materials have been removed almost completely. Considering that the thickness of PS shell of the hollow capsule is $33 \mathrm{~nm}$, the above result of $T_{\mathrm{g}}$ may be surprising which is remarkably different from that for PS freestanding thin films: reduction in $T_{\mathrm{g}}$ of at most several tens of $\mathrm{K}$ from the bulk value has been reported for PS free-standing thin films of $30 \mathrm{~nm}$ in thickness. ${ }^{9}$ On the other hand, $T_{\mathrm{g}}$ has been reported to increase from the bulk value for PMMA thin films formed on a silicon oxide substrate, which has been interpreted to be due to a strong interaction with the substrate at the interface. ${ }^{11,20}$ PMMA/BS-P and PMMA hollow show almost the same $T_{\mathrm{g}}$ (bulk $T_{\mathrm{g}}$ ), thus, for PMMA/BS$\mathrm{P}, T_{\mathrm{g}}$ may not be influenced by the interface between PMMA and BS-P.

From the above results, we conclude that the present capsules of both PMMA and PS show $T_{\mathrm{g}}$ almost identical to their bulk values. Similar result has been found for PS microspheres with diameters of less than $100 \mathrm{~nm} .{ }^{12}$ In this respect, the present polymeric capsules show thermal properties similar to a spherical particle rather than to a thin film, suggesting that the polymer chain configurations of the capsules are similar to those of a microsphere rather than to a thin film. However, it may be difficult to reconcile the present results of $T_{\mathrm{g}}$ with those obtained for thin films and microspheres simply by their geometric differences. Further investigations on nano-sized polymer materials with various geometries should be done to resolve this

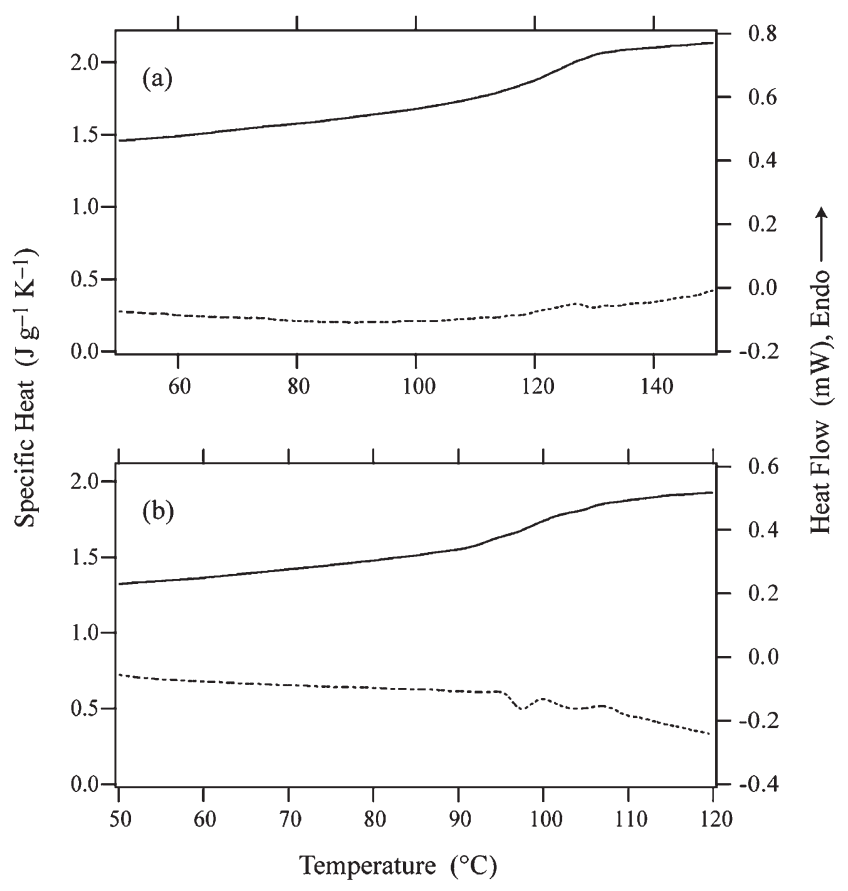

Figure 4. DSC traces obtained from the step-scan mode for (a) PMMA hollow and (b) PS hollow. The solid curves indicate reversing specific heat, and the dotted curves indicate non-reversing heat flow.

problem.

Figure 4 illustrates DSC traces obtained from the step-scan mode. Non-reversing heat flow for PMMA hollow shows a slight endotherm around $T_{\mathrm{g}}$ which is an enthalpy overshoot due to structural relaxation below $T_{\mathrm{g}}$ (physical aging). On the other hand, PS hollow shows a somewhat complicated curve of non-reversing heat flow around $T_{\mathrm{g}}$. The curve may contain exotherms for certain structural relaxation during the scan as well as the enthalpy overshoot (endotherm). Such structural relaxations are probably associated with a morphological change from the fine hollow particles to the bulk state, whereby the large surface area of the hollow particle is lost. Two or three exotherms are observed around $T_{\mathrm{g}}$ for PS hollow, but the origin of this multiple exotherm is not clear. From the total area of these exotherms, we roughly estimated that $54 \%$ of the surface of PS hollow capsules was lost through these exothermic processes. In this estimation, we used the literature value of $35 \times 10^{-3} \mathrm{~J} \mathrm{~m}^{-2}$ for the surface free energy of PS at $373 \mathrm{~K} .{ }^{21}$ As for PMMA hollow, such morphological change is not suggested from the observed DSC trace, but visual observation revealed that the sample after a DSC scan up to $200^{\circ} \mathrm{C}$ was completely bulk-like (the powder was apparently melted). It is likely that for PMMA hollow, the structural relaxation from a hollow capsule to a bulk state upon heating occurs so slowly that no apparent exotherm is observed. This slow relaxation for PMMA hollow might be partially due to its higher 
molecular weight than for PS hollow (see Table I).

Surface coverage of the whisker particles with the polymer shell can be roughly estimated from the dimensions of the particles (Table II) and $\Delta C_{p}$ values (Table III). The obtained values are $150 \%$ for PMMA/BS-P, and $120 \%$ for PS/BS-P. These values of exceeding $100 \%$ may be because of the broad distribution of both the layer thickness and dimensions of the particles as shown in Table II, but the result at least suggests that the surface of BS-P has been covered with the polymer shell fairly well. In the present emulsion polymerization, we added a small amount of methacrylic acid 3-(trimethoxysilyl)propyl ester and 2-hydroxymethyl methacrylate. These reagents are expected to couple with hydroxyl groups on the surface of BS-P that have been treated with phosphoric acid. With the latter reagent, a condensation reaction may occur resulting in the formation of (polymer)-Si-O(BS-P) linkage. The molar ratios of the two reagents with respect to the monomer affect the surface coverage of the polymer, ${ }^{14}$ and in this study, the preparations were done with the optimized ratios that provide good surface coverage.

\section{CONCLUSIONS}

In this paper, we have demonstrated that polymeric capsules can be successfully prepared by using calcium carbonate whisker as a template core. Hollow particles can also be prepared by dissolving the core with hydrochloric acid. The thickness of the outer polymer shell was very thin (less than $100 \mathrm{~nm}$ ). DSC measurements have revealed that these very thin polymer shell materials of both PMMA and PS show $T_{\mathrm{g}}$ 's very similar to those of the bulk samples. This may be surprising compared with the results of $T_{\mathrm{g}}$ reduction reported for thin polymer films with similar thicknesses. Dimensions (diameter and thickness) of the obtained capsules in the present study have fairly broad distributions. We are planning to refine the preparation method in order to yield narrower size distributions.

Acknowledgment. The authors thank Mr. Hidemitsu Kasahara and Mr. Koji Kobayashi, Maruo Calcium Co., Japan, for supplying the calcium carbonate whisker, and for their helpful suggestions for sample preparations. The authors thank Prof. Kazunori Se for help in the molecular weight determination by size exclusion chromatography.

\section{REFERENCES}

1. K. B. Thurmond II, T. Kowalewski, and K. L. Wooley, J. Am. Chem. Soc., 119, 6656 (1997).

2. A. Harada and K. Kataoka, Science, 283, 65 (1999).

3. B. Miksa and S. Slomkowski, Colloid Polym. Sci., 273, 47 (1995).

4. J.-E. Jönsson, O. J. Karlsson, H. Hassander, and B. Törnell, Macromolecules, 34, 1512 (2001).

5. I. L. Radtchenko, G. B. Sukhorukov, S. Leporatti, G. B. Khomutov, E. Donath, and H. J. Möhwald, Colloid Interface Sci., 230, 272 (2000).

6. D. G. Shchukin, I. L. Radtchenko, and G. B. Sukhorukov, J. Phys. Chem. B, 107, 86 (2003).

7. C. L. Jackson and G. B. McKenna, J. Non-Cryst. Solids, 131-133, 221 (1991).

8. K. Fukao and Y. Miyamoto, Phys. Rev. E, 61, 1743 (2000).

9. J. Mattsson, J. A. Forrest, and L. Borjesson, Phys. Rev. E, 62, 5187 (2000).

10. a) J. L. Keddie, R. A. L. Jones, and R. A. Cory, Europhys. Lett., 27, 59 (1994).

b) J. L. Keddie, R. A. L. Jones, and R. A. Cory, Faraday Discuss., 98, 219 (1994).

11. D. S. Fryer, P. F. Nealey, and J. J. de Pablo, Macromolecules, 33, 6439 (2000).

12. U. Gauer and B. Wunderlich, Macromolecules, 13, 1618 (1980).

13. T. Sasaki, A. Shimizu, T. H. Mourey, C. T. Thurau, and M. D. Ediger, J. Chem. Phys., 119, 8730 (2003).

14. K. Kobayashi and H. Kasahara, Jpn. Patent 98164 (2001).

15. 2-Hydroxymethyl methacrylate and methacrylic acid 3-(trimethoxysilyl)propyl ester were added in order to improve the compatibility between the polymer and BS-P. See also ref 14.

16. B. Cassel, Am. Lab., 32, 23 (2000).

17. T. Sasaki, N. Yamauchi, S. Irie, and K. Sakurai, J. Polym. Sci., Part B: Polym. Phys., 43, 115 (2005).

18. T. Motoyama and S. Okamura, Kobunshi Kagaku, 12, 108 (1955).

19. I. Piirma, Ed., "Emulsion Polymerization," Academic Press, New York, N.Y., 1982.

20. D. S. Fryer, R. D. Peters, E. J. Kim, J. E. Tomaszewski, J. J. de Pablo, P. F. Nealey, C. C. White, and W. Wu, Macromolecules, 34, 5627 (2001).

21. J. Brandrup, E. H. Immergut, E. A. Grulke, and D. Bloch, Ed., "Polymer Handbook," 4th ed., John Wiley-Interscience, New York, N.Y., 1999. 